\title{
On the influence of CMEs on the global 3-D coronal electron density
}

\author{
M. Kramar ${ }^{1,2}$, J. Davila ${ }^{2}$, H. Xie ${ }^{1,2}$, and S. Antiochos ${ }^{2}$ \\ ${ }^{1}$ Physics Department, The Catholic University of America, 620 Michigan Ave NE, Washington, D.C. 20064, USA \\ ${ }^{2}$ NASA Goddard Space Flight Center, Greenbelt, MD 20771, USA
}

Received: 11 June 2009 - Revised: 18 February 2011 - Accepted: 18 May 2011 - Published: 15 June 2011

\begin{abstract}
In order to analize the influence of a Coronal Mass Ejection (CME) on the coronal streamer belt, we made 3-D reconstructions of the electron density in the corona at heliospheric distances from 1.5 to $4 R_{\odot}$ for periods before and after a CME occured. The reconstructions were performed using a tomography technique. We studied two CME cases: (i) a slow CME on 1 June 2008; (ii) two fast CMEs on 31 December 2007 and 2 January 2008. For the first case of slow CME, it was found: (i) the potential magnetic field configuration in the CME initiation region before the CME does not agree with the coronal density structure while after the CME the agreement between the field and density is much better. This could be manifistation of that that the field was non-potential before the CME and after the CME the field relaxes towards a more potential state. (ii) It was shown that the dimming caused by the slow CME is not due to rotation of the corona and a line-of-sight (LOS) effect but a streamer blow out effect took place.
\end{abstract}

Keywords. Solar physics, astrophysics, and astronomy (Corona and transition region; Flares and mass ejections; Magnetic fields)

\section{Introduction}

Coronal Mass Ejections (CME) plays an important role for space weather research. There are still many open questions connected with CME initiation, propagation and influence on the ambient corona (Hudson et al., 2006; Mikic and Lee, 2006). Recent research has revealed, however, that CMEs involve the release of the magnetic energy stored in magnetic field (Forbes, 2000; Klimchuk, 2001). So, analyzing the magnetic field could help to understand the nature of

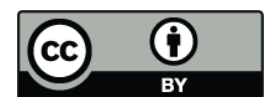

Correspondence to: $\mathrm{M}$. Kramar (kramar@helio.gsfc.nasa.gov)
CMEs. But for the moment our knowledge about the coronal magnetic field is very limited. The direct Zeeman and Hanle effect (usually used for deriving the magnetic field at the photosphere) measurements in the corona are very difficult due to much lower strength of the coronal magnetic field (about 1 Gauss (Lin et al., 2004)) and because of the high temperature of the coronal plasma. Moreover, the interpretation of such kind of measurements is not straightforward due to the fact that the corona is opticaly thin and these measurements are essentially line-of-sight (LOS) integrated. However the latest progress in these measurements (Lin et al., 2004; Tomczyk et al., 2008; Judge, 2007) and the vector tomography technique (Kramar et al., 2006; Kramar and Inhester, 2007) gives a hope for increasing our knowledge about the coronal magnetic field.

Recently, vector magnetograms from the photosphere have became available, which in principle supply all the information necessary for a non-linear force-free field extrapolation of the surface data into the solar corona (Wiegelmann et al., 2005; Wiegelmann, 2008). The realistic force-free field extrapolation, however, is highly ill-posed and yields less reliable results, the larger the distance from the surface and the stronger the currents (Demoulin et al., 1992).

For the moment, a robust way to estimate the coronal field is to extrapolate the measured photospheric LOS field by using the potential field approximation (Altschuler and Newkirk, 1969). Since the potential magnetic field is the field with the minimum energy for a given photospheric radial boundary condition (Sakurai, 1989), it cannot account for dynamical processes such as eruptions, flares, magnetic reconnections, where very probably magnetic energy is converted into plasma kinetic energy without a significant change regarding the magnetic boundary flux on the same fast time scale. Indeed, soft X-ray observations of active regions often show a non-potential structure of the magnetic field (Jiao et al., 1997).

Published by Copernicus Publications on behalf of the European Geosciences Union. 
In the present paper we study the pre- and post-CME coronal electron density structure and compared it with the potential field source surface (PFSS) models for the correspondent periods. We considered two CME cases belonging to different types. One case consists of a slow CME, which probably originated relatively high in the corona, and the second case consists of two fast CMEs having source regions close to the Sun's surface in the same active region. The comparison of the pre- and post-CME states of the corona for these two different types of CME could provide us additional knowledge for better understanding of how a CME influences the coronal streamer belt, and about the propagation and initiation mechanism of CMEs as well. The reconstructions of the coronal electron density were performed using a tomography technique.

\section{Tomography}

The solar corona is optically thin, so in coronagraph images the radiation coming from the corona is integrated over the observer's line-of-sight (LOS), and it is impossible to localize any structure in the corona with an observation from only one viewing direction. To reconstruct extended structures in the optically thin corona, it is necessary to have observations from more than two directions. This is the essence of tomography. In practice, a rigid rotation of the coronal density structures is usually assumed. Coronagraph data from half a solar rotation are necessary as input for the reconstruction algorithm, and only structures which are stationary over about 14 days can reliably be reconstructed (Davila, 1994; Zidowitz, 1999; Frazin and Kamalabadi, 2005; Kramar et al., 2009). Here we use the regularized tomography method where the regularization is in the form of first-order smoothing term.

For our density reconstructions we use the $p B$-intensity images from COR1 instrument onboard the Solar Terrestrial Relations Observatory B (STEREO-B) taken approximately 2.3 times per day as input for the tomographic inversion. Because it views the corona close to the limb, the COR1 instrument has significant amount of scattered light that must be subtracted from the image prior to reconstruction. Proper removal of instrumental scattered light is essential for coronal reconstruction. One of the ways to do this is to subtract a monthly minimum (MM) background. The monthly minumum approximates the instrumental scatter by finding the minimum value of each pixel in all images over roughly a one month period. However, this method tends to overestimate the scattered light in the streamer belt (equatorial region). For these pixels, their minimal value over a month will contain both the scattered light and the steady intensity value from the corona. Hence, using such pixels as input for electron density reconstruction, we would obtain an electron density which is lower than the actual density.
Another way to remove the scattered light is to subtract a roll minimum (RM) background. The roll minimum background is the minimum value of each pixel obtained during roll maneuver of the spacecraft (instrument) around its optical axis. Because the coronal polar regions are much darker than equatorial ones, the minimum value of pixels in the equatorial region during the roll maneuver are nearer to the value of the scattered light intensity than the MM.

The sensitivity of the instrument changes with time decreasing about $0.25 \%$ per month (Thompson and Reginald, 2008). Also, the distance of the spacecraft from the Sun changes causing changes in the scattered light. But the roll maneuvers are done rather rarely. Therefore it is impossible to use a RM background made in one month for data from other month when maximum photometric accuracy is needed. One of the ways to get a background image for the period between the roll maneuveres, is to interpolate RM backgrounds over the time in a such way that this time dependence follows time dependence of the MM backgrounds as the MM background images available for every month. This approach to get background image is realized by W. Thompson in SolarSoft IDL routine secchi prep with keyword parameter calroll. We used backgrounds obtained in this way. The photometric calibration is based on Jupiter passage through COR1 FOV (Thompson and Reginald, 2008).

After subtracting the scattered light, a median filter with the width size of 3 pixels was applied in order to reduce anomalously bright pixels caused by cosmic rays. Then, every third image pixel was taken (resulting in a $340 \times 340$ pixel image) in order to reduce used computer memory size. Since the recontruction domain is rectangular with a size of $128^{3}$ covering $4 R_{\odot}$ sphere, this input image size does not significantly influence on the reconstruction results.

The inversion is performed for the function

$F=|\mathbf{A} \cdot \mathbf{X}-\mathbf{Y}|^{2}+\mu|\mathbf{R} \cdot \mathbf{X}|^{2}$.

Here, the elements $x_{j}$ of the column matrix $\mathbf{X}$ contain the values of electron density $N_{\mathrm{e}}$ in the grid cells with index $j=1, \ldots, n$, and $y_{i}$ is the data value for the $i$-th ray, where in$\operatorname{dex} i=1, \ldots, m$ accounts for both the viewing direction and pixel position in the image. The matrix element $a_{i j}$ represents the intersection of volume element $j$ with LOS related to the pixel $i$, multiplied by the kernel function that defined by the Thompson scattering effect for $p B$-intensity signal (Van de Hulst, 1950; Billings, 1966; Quemerais and Lamy, 2002; Kramar et al., 2009). The second term on the righthand side of Eq. (1) is the regularization term used in order to minimize the effects of noise and data gaps (Tikhonov, 1963). The matrix $\mathbf{R}$ is a diagonal-like matrix such that the regularization is the first order smoothing term, i.e. the square difference in value between two neighboring grid cells, summed over all cells. The regularization parameter, $\mu$, regulates balance between the smoothness of the solution on one hand, and the noise and reconstruction artifacts on other. The result of the inversion depends on a number of factors, including 
Table 1. Observation periods of input images for the tomographic reconstructions, and masses of the CMEs and streamer belt losses in the CME impacted regions (areas bounded by white square and round lines in Figs. 1, 3, 5, 7; also see Sect. 3 of the text).

\begin{tabular}{|c|c|c|c|c|c|c|}
\hline & \multicolumn{2}{|c|}{ reconstruction for pre CME corona } & \multicolumn{2}{|c|}{ reconstruction for post CME corona } & \multicolumn{2}{|c|}{$\operatorname{mass}[\mathrm{g}]$} \\
\hline & start obs. $/ \phi_{\text {LOS }}$ & end obs./ $\phi_{\text {LOS }}$ & start obs. $/ \phi_{\mathrm{LOS}}$ & end obs./ $\phi_{\text {LOS }}$ & CME & $\begin{array}{l}\text { streamer } \\
\text { loss }\end{array}$ \\
\hline CME1 & $\begin{array}{l}2008-05-17 \text { T00:20 } \\
290^{\circ}\end{array}$ & $\begin{array}{l}2008-05-31 \mathrm{~T} 23: 45 \\
92^{\circ}\end{array}$ & $\begin{array}{l}2008-06-03 \mathrm{~T} 01: 15 \\
64^{\circ}\end{array}$ & $\begin{array}{l}2008-06-16 \mathrm{~T} 13: 00 \\
245^{\circ}\end{array}$ & $0.9 \times 10^{15}$ & $0.98 \times 10^{15}$ \\
\hline CME2ab & $\begin{array}{l}2007-12-17 \mathrm{~T} 13: 05 \\
130^{\circ}\end{array}$ & $\begin{array}{l}2007-12-30 \mathrm{~T} 23: 45 \\
312^{\circ}\end{array}$ & $\begin{array}{l}2008-01-03 \mathrm{~T} 08: 45 \\
267^{\circ}\end{array}$ & $\begin{array}{l}2008-01-16 \mathrm{~T} 14: 25 \\
93^{\circ}\end{array}$ & $\begin{array}{l}4.3 \times 10^{15} \\
(2 a) \\
1.1 \times 10^{15} \\
(2 b)\end{array}$ & $1.1 \times 10^{15}$ \\
\hline
\end{tabular}

the number of iterations, and value of $\mu$. The value of $\mu$ was chosen using the cross-validation method (Frazin and Janzen, 2002). The iterations are performed until the first term in Eq. (1) becomes slightly less than the data noise level which is essentially the Poisson noise in the data.

In order to increase the contribution of signals from those LOS which pass through the low density regions, and to reduce the artifacts in the numerical reconstruction at larger distances from the Sun, a weighting function (or preconditioning) was applied. The regularization parameter and number of iterations were chosen by cross-validation method. Detailed describtion of the used tomography method can be found in Kramar et al. (2009). As an output from the inversion we have 3-D electron density reconstruction of the corona at heliospheric distances from 1.5 to $4.0 R_{\odot}$.

Preliminary error analysis shows that the error due to sparseness of the data (2-3 per day) and regularization is of order $10 \%$, and the error due to non-stationarity of the corona during the period of observations (typically 14 days) could be up to $50 \%$. More detailed analysis of the error for the method is to be presented in the forthcoming paper.

\section{Pre- and post-CME coronal streamer belt structure}

We analized pre- and post-CME coronal streamer belt structure for two CME cases:

1. slow CME on 1 June 2008 (CME1).

2. two fast CMEs on 31 December 2007 (CME2a) and 2 January 2008 (CME2b).

The source regions of these two fast CMEs are very close to each other and located in the same active region near the Sun's surface. Taking also into account that these CMEs occured within a time interval of two days, we join them in one case study.

To reconstruct the 3-D electron density for pre- and postCME corona, we used STEREO-B/COR1 data collected during a half of the solar rotation period just before and after a CME. The starting and ending observation dates and corresponded Carrington longitudes of STEREO-B spacecraft, $\phi_{\text {LOS }}$, are summarized in Table 1 . Note, that the longitude decreases during the observations.

\subsection{CME on 1 June 2008 (CME1)}

The CME1 is a slow CME. The two most characteristic features found for this CME are (Robbrecht et al., 2009): (i) it contains a significant mass, and (ii) it was not found clear on-disk signatures to be identified as a source region for this CME (Robbrecht et al., 2009).

As the CME1 happened on 1 June 2008, to reconstruct the 3-D electron density in the corona before and after the CME, we used STEREO-B/COR1 data collected during a half of the solar rotation period just before and after the CME, i.e. during 17-31 May 2008 for the pre-CME density reconstruction, and during 3-16 June 2008 for the post-CME density reconstruction (see Table 1). The spherical cross-sections of the reconstructed electron densities at heliocentric distances 2.0, 2.2 and $2.4 R_{\odot}$ are presented in Fig. 1. The independent localizations of the CME (Robbrecht et al., 2009) by several methods, indicates that the density decreases significantly in the streamer belt near Carrington longitude of $80^{\circ}$ is caused by the CME.

This region was in plane of the sky (POS) position for STEREO-B spacecraft on 26 May at 01:50 UT (east limb) and 8 June at 15:12 UT (west limb). So, the pre- and postCME density reconstructions of the CME1 coronal region at $80^{\circ}$ longitude reflects state of the corona at times about 4-5 days before and 7-8 days after the CME (STEREO-B for shifts for about $13.3^{\circ}$ in longitude per day during the observational period). So, as seen from Figs. 1 and 2, the post-CME density remains significantly reduced in comparison with pre-CME state for at least about 7-8 days in the CME region.

Figure 3 shows a difference in reconstructed electron density for the periods after the CME1 and before. It is seen that the density mainly decreased after the CME in a region in the corona within Carrington longitudes from 60 to 

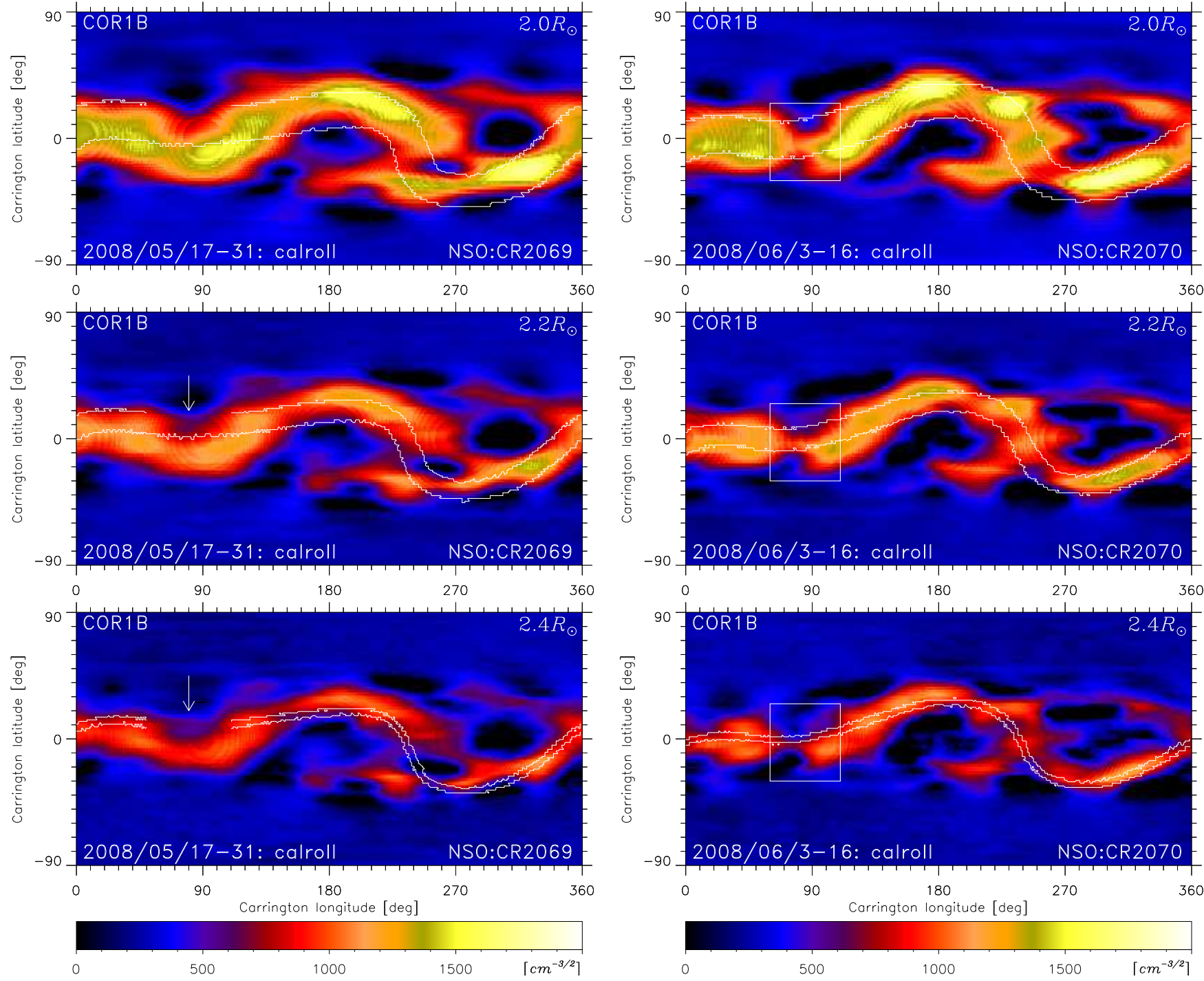

Fig. 1. Spherical cross-section of the reconstructed electron density in square root scale at heliocentric distances $2.0,2.2$ and $2.4 R_{\odot}$ (the distances are shown in the right upper corners). The reconstruction for the period before the CME of 1 June 2008 is shown on the left panel while the reconstruction for the period after this CME is shown on the right panel. The white lines are the boundaries between closed and open magnetic field lines for PFSS model with source surface at $2.5 R_{\odot}$ corresponded for CR 2069 and CR 2070, i.e. periods approximately before and after the CME. The part of the lines on the left panel between Carrington longitudes 50 and $110^{\circ}$ are not shown in order to more clearly show the connecting structure in this region (marked with arrow). However the lines in this region are almost horizontal.

$110^{\circ}$, latidudes from -30 to $25^{\circ}$, and heliocentric distance from 1.5 to $3.6 R_{\odot}$. As the reconstructions gives the electron density in physical units, we can calculate the mass lost by the corona within this region. So, assuming $10 \%$ helium abundance which corresponds to mass per electron number equal to $1.974 \times 10^{-24} \mathrm{~g}$ (routine ne2mass in SolarSoft IDL library), we found the mass lost is $9.8 \times 10^{14} \mathrm{~g}$. The mass of the CME measured in COR1 FOV is $\sim 8 \times 10^{14} \mathrm{~g}$ (see appendix for description). The maximal mass of the CME measured in COR1 FOV estimated by Robbrecht et al. (2009) has about the same value of $\sim 9 \times 10^{14} \mathrm{~g}$. This fact is evidence that material the CME consists of could be originated mainly from the streamer belt. However, it is difficult to make a final conclusion about the origin of the CME's material because our estimation of the mass loss of the streamer is based on the reconstructions of the corona from $1.5 R_{\odot}$. So, we do not know the pre- and post CME density below $1.5 R_{\odot}$.

The STERO-B/COR1 data for the tomography reconstruction the post-CME corona which mainly contains information from the region where CME occured is collected about a week after the CME because the CME was directed approximately towards to STEREO-B. So, during this period the 


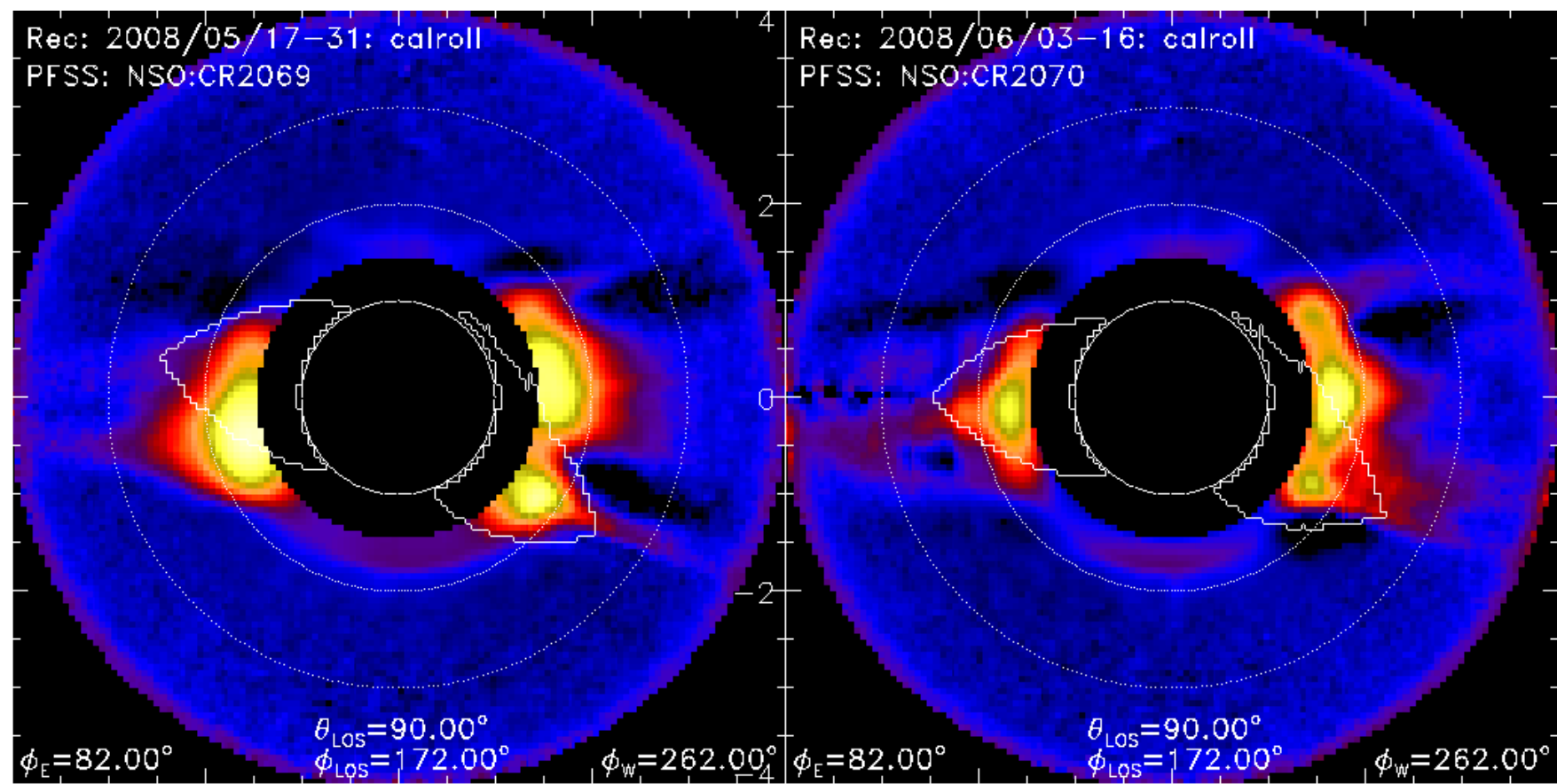

Fig. 2. Cross-sections of the reconstructed electron density in square root scale by a plane perpendicular to a LOS with Carrington longitude of $172^{\circ}$ and colatidude of $90^{\circ}$. The reconstruction for the period before a CME of 1 June 2008 is shown on the left side while the reconstruction for the period after this CME is shown on the right side. The white contour lines are the boundaries between closed and open magnetic field lines for a PFSS model with source surface at $2.5 R_{\odot}$ for CR 2069 and CR 2070, i.e. periods approximately before and after the CME. The white circles mark heliospheric distances for 1,2 and $3 R_{\odot}$.
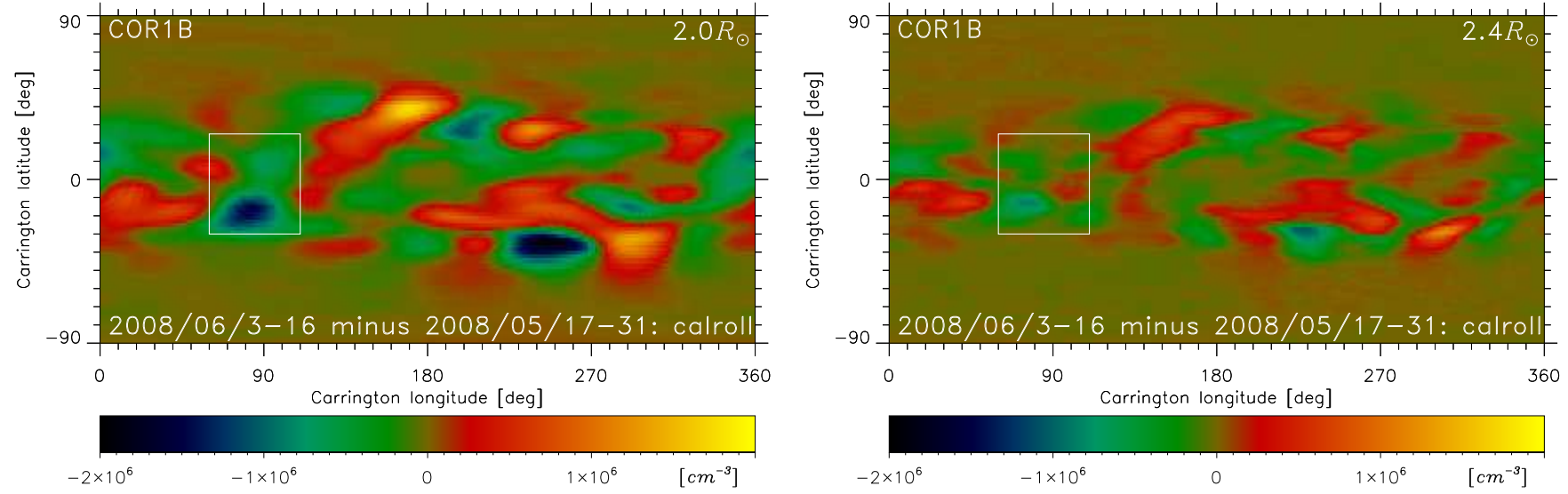

Fig. 3. Difference in reconstructed electron density for the periods after the CME of 1 June 2008 (CME1) and before. The spherical cross-sections are shown at heliocentric distances 2.0 and $2.4 R_{\odot}$ (the distances are shown in the right upper corners).

streamer belt could be partially filled by the plasma. Therefore our estimation of mass lost by the streamer belt could be a lower limit.

The white contour lines in Fig. 1 represent the boundaries between closed and open magnetic field lines in potential field source surface model (PFSS) with the source surface located at $2.5 R_{\odot}$ for the Carrington rotations $(\mathrm{CR})$ correspond to pre- and post-CME times, i.e. CR 2069 and CR 2070, re- spectively. The PFSS model is based on NSO/GONG data. The harmonic coefficients in the PFSS model are restricted to ninth order which is satisfactory for the coronal scale used in this study. It is clearly seen that the PFSS model do not match with the streamer belt in the CME region before the CME while after the CME the PFSS model and the streamer belt in the CME region are in significantly better agreement. 

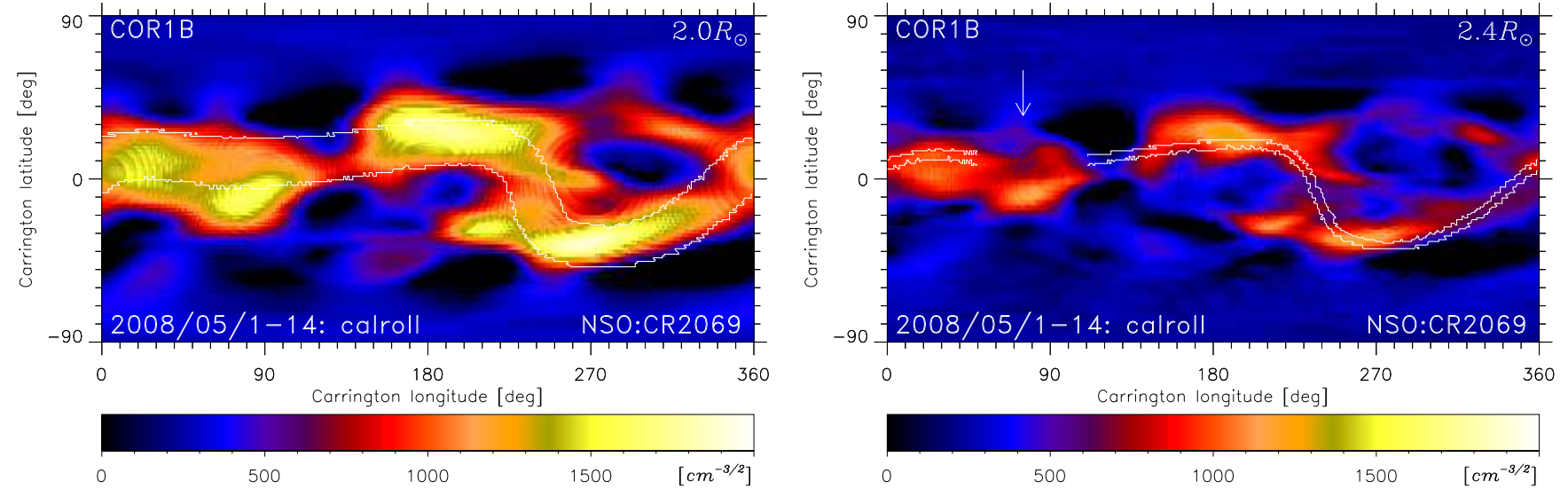

Fig. 4. Spherical cross-section of the reconstructed electron density in square root scale at heliocentric distances 2.0 and $2.4 R_{\odot}$ (the distances are shown in the right upper corners). The reconstruction based on data obtained during 1-14 May 2008. The white lines are the boundaries between closed and open magnetic field lines for PFSS model with source surface at $2.5 R_{\odot}$ corresponded for CR 2069 . The part of the lines on the right panel between Carrington longitudes 50 and $110^{\circ}$ are not shown in order to more clearly show the density structure in this region. However the lines in this region are almost horizontal.

The PFSS reconstructions are based on the magnetograph observations from the Earth. The NSO/GONG observations for CR 2069 ended on 2008-05-20T23:54 when Earth was at $262^{\circ}$ longitude, and for CR 2070 on 2008-06-16T23:54 when Earth was at $265^{\circ}$ longitude. The Earth was at $80^{\circ}$ longitude (CME1 region) on 2008-05-07T12:40 corresponding to CR 2069 and 2008-06-03T17:47 corresponding to CR 2070.

On 2008-06-03T17:47, just after the CME1, the Earth was at $80^{\circ}$ longitude (CME1 region). This date corresponds to CR 2070. Therefore we compare PFSS model for CR 2070 with the post-CME reconstruction. And this comparison has sence for longitudes around the CME1 region, but could be not valid for the rest of the corona because of the difference in the observation dates for data needed for the PFSS model and tomographic reconstruction.

The magnetograph data for the pre-CME1 period is obtained during about 2008-05-07T12:40 date corresponding to CR 2069. But presented on Figs. 1 and 2 the density reconstruction for the pre-CME1 period is based on data obtained during 17-31 May 2008, which is more than a week after the central meridian passage through CME1 region. Therefore it is useful to make another reconstruction for period of 1-14 May 2008 in order to look how the corona changes. Figure 4 shows the spherical cross-sections of the reconstructed electron densities at heliocentric distances 2.0 and $2.4 R_{\odot}$ when input COR1B images for the inversion are from 1-14 May 2008. We can see similar density structure near Carrington longitude of $80^{\circ}$ as for the 17-31 May reconstruction.

Figure 2 represents cross-sections of the reconstructed electron density by a plane perpendicular to a LOS with Carrington longitude of $172^{\circ}$ and latidude of $0^{\circ}$. The reconstruction for the period before a CME of 1 June 2008 is shown on the left side while the reconstruction for the period after this
CME is shown on right side. The east sides from the Sun correspond to the region with Carrington longitude of $82^{\circ}$, i.e. where the CME1 took place. We see that the streamer shape after the CME is significantly reduced in height in contrary with much less evidence of the shape reducing in latitudinal direction. This could be indication that the area in the Sun's surface, where the closed magnetic field lines originate does not change after the CME eruption. To get more insights we would have to carry out a tomographic reconstruction of the corona below $1.5 R_{\odot}$ that could be based on data from Mauna Loa Solar Observatory (MLSO) Mark-IV coronameter.

\subsection{CMEs on 31 December 2007 and 2 January 2008}

To reconstruct the 3-D electron density in the corona before and after the CMEs, we used STEREO-B/COR1 data collected during a half of the solar rotation period just before and after the CMEs, i.e. during 17-30 December 2007 for the pre-CME density reconstruction, and during 3-17 January 2008 for the post-CME density reconstruction (Table 1). The spherical cross-sections of the reconstructed electron densities at heliocentric distances 2.0, 2.2 and $2.4 R_{\odot}$ are presented in Fig. 5. The CME locations were determined by performing 3-D triangulations from simultaneous STEREO A and B images of SECCHI/COR1 using the IDL SolarSoft routine scc-measure, and then it has been verified with SEC$\mathrm{CHI} / \mathrm{EUVI}$ images if the source locations of the CME appear on solar disk in either STEREO A and B. By this means, it was found that the source regions of both CMEs are located near Carrington longitude of $235^{\circ}$. This region was in plane of the sky (POS) position for STEREO-B spacecraft on 29 December at 23:53 UT (east limb) and 12 January at 15:00 UT (west limb). So, the pre- and post-CMEs density reconstructions of the CME2ab coronal region at $235^{\circ}$ 

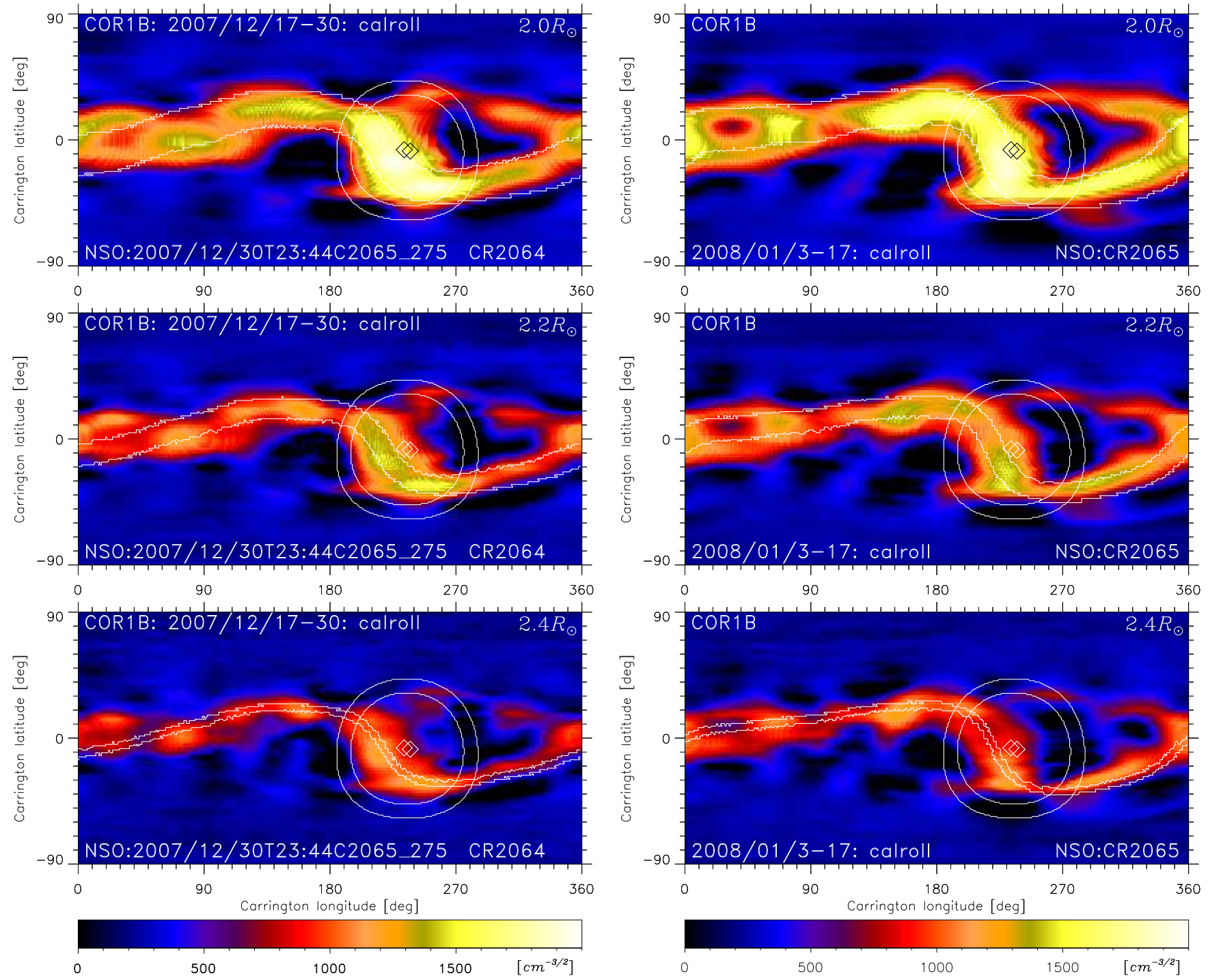

Fig. 5. Spherical cross-section of reconstructed electron density in square root scale at heliocentric distances $2.0,2.2$ and $2.4 R_{\odot}$ (the distances are shown in the right upper corners). The reconstruction for the period before CMEs of 31 December 2007 and 2 January 2008 is shown on the left panel while the reconstruction for the period after these CMEs is shown on the right panel. The white lines are the boundaries between closed and open magnetic field lines for a PFSS model with source surface at $2.5 R_{\odot}$ for CR 2064 and CR 2065 , i.e. periods approximately before and after the CMEs. Two rombs indicate the source regions for CME2ab. Circles shows the region used for the computation of the mass loss.

Carrington longitude reflects state of the corona at times about a day before and 7-12 days after the CMEs (STEREO$\mathrm{B}$ shifts for about $13.2^{\circ}$ in longitude per day during the observational period).

The NSO/GONG observations for CR 2064 PFSS model ended on 5 January 2008 23:34 UT when Earth was at $256^{\circ}$ longitude, and for CR 2065 on 1 February 2008 23:44 UT when Earth was at $260^{\circ}$ longitude. The Earth was at $235^{\circ}$ longitude (CME2ab region) on 11 December 2007 05:05 UT corresponding to CR 2064 and 7 January 2008 13:00 UT corresponding to CR 2065.
As integral NSO/GONG harmonic coefficients for CR 2064 at about $235^{\circ}$ longitude are based on observations both before and after CME2ab date, for the PFSS model for the pre-CME period we used the harmonic coefficients based on observations finished on 30 December 2007 23:44 UT when Earth was at $334^{\circ}$ longitude.

Figure 7 shows a difference in reconstructed electron density for the periods after the CME2ab and before. We selected the axisymmetric conic region in the corona where the angular position of the cone's axis is at Carrington longitude of $235^{\circ}$ and latidude of $-7.5^{\circ}$, and the cone's apex angle of $100^{\circ}$ (the boundary of the cone is marked by a largest nearly 


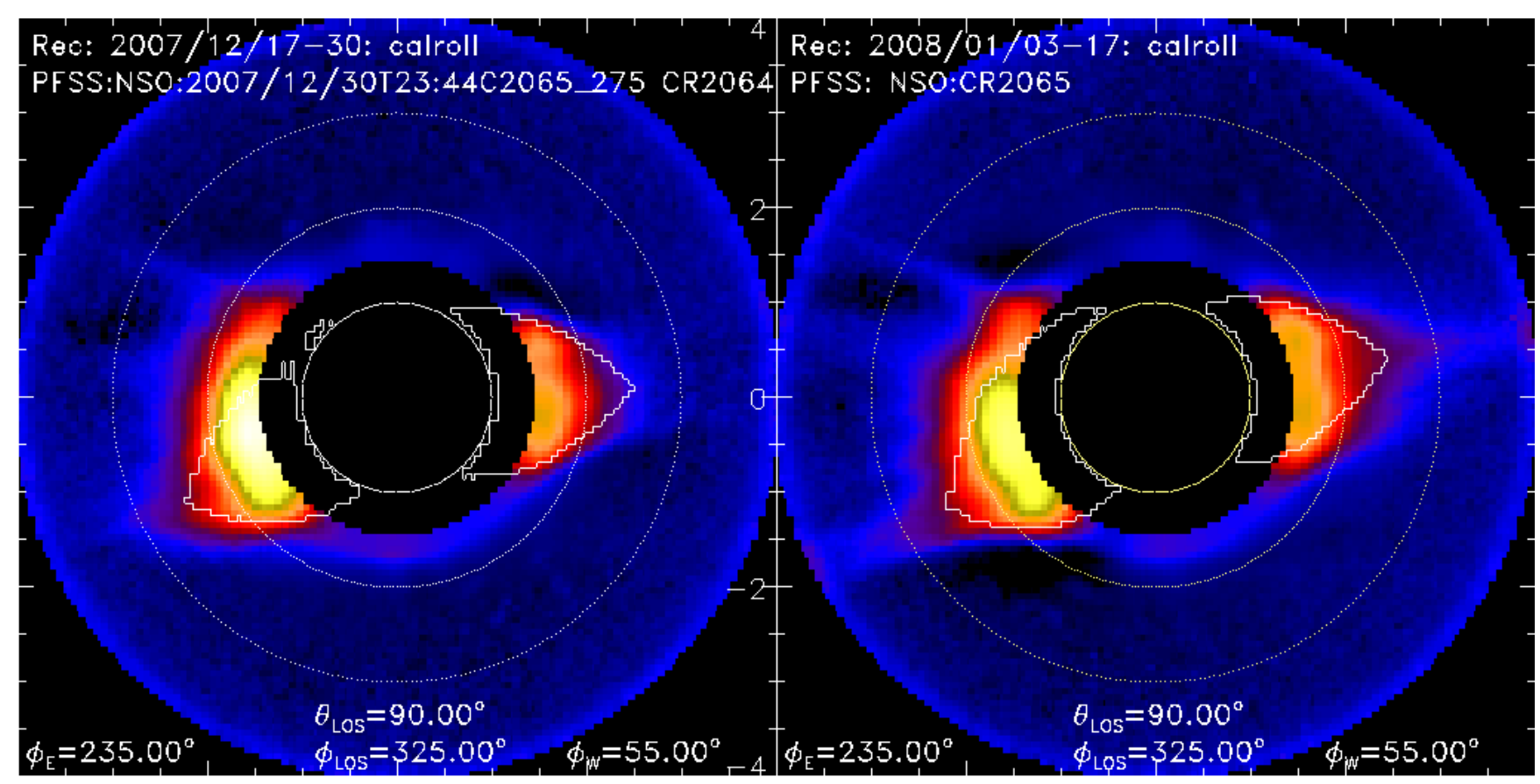

Fig. 6. Cross-sections of the reconstructed electron density in square root scale by a plane perpendicular to a LOS with Carrington longitude of $325^{\circ}$ and colatidude of $90^{\circ}$. The reconstruction for the period before the CME2ab is shown on the left side while the reconstruction for the period after CME2ab is shown on the right side. The white contour lines are the boundaries between closed and open magnetic field lines for a PFSS model with source surface at $2.5 R_{\odot}$ for CR 2064 and CR 2065, i.e. periods approximately before and after the CME. The white circles mark heliospheric distances for 1,2 and $3 R \odot$.
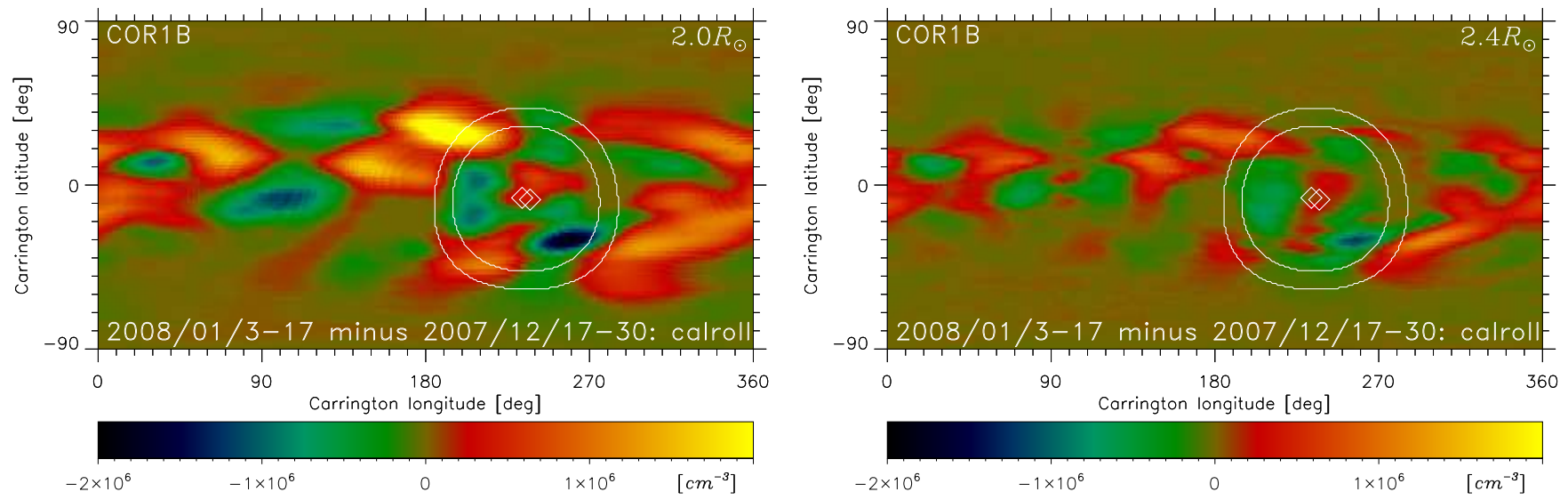

Fig. 7. Difference in reconstructed electron density for the periods after and before the CMEs of 31 December 2007 (CME2a) and 2 January 2008 (CME2b). The spherical cross-sections are shown at heliocentric distances 2.0 and $2.4 R_{\odot}$ (the distances are shown in the right upper corners).

circle line in Figs. 5 and 7) We can estimate the mass lost by the corona in the region within this cone and heliocentric distances from 1.5 to $3.6 R_{\odot}$. Assuming $10 \%$ of helium abundance, which corresponds to mass per electron number equal to $1.974 \times 10^{-24} \mathrm{~g}$ (routine ne2mass in SolarSoft IDL library), we found a mass loss of $1.1 \times 10^{15} \mathrm{~g}$. Note, that this number is a mass difference within the selected region, i.e. it takes into account also increase of the mass for whatever reason caused by the CMEs or not . The only negative part of the difference gives a number of $2.5 \times 10^{15} \mathrm{~g}$.

It was found that the masses of CME2a and CME2b in the COR1 FOV are $4.3 \times 10^{15}$ and $1.1 \times 10^{15} \mathrm{~g}$, respectively, which are comparable or more than the mass of CME1 in COR1 FOV. In contrary with CME1 case, the estimated 
streamer belt mass loss for CME2ab case is less than total masses of CME2ab. Moreover, the PFSS model for the period before CME2ab is in much better agreement with the reconstructed electron density structure than the PFSS model for the period before CME1. So, these properties could be explained that CME2ab has source region deep in the corona near the photosphere which is clearly seen in EUVI 304 and during their expansion the CME2ab "pushed out" surrounding materia. However, the lower number of mass loss could also be explained by the fact that the CME2ab originates in an active region with higher density and after the CMEs the density above this active region was "recovered" faster than time needed to collect data for tomographic reconstruction.

Figure 6 represents cross-sections of the reconstructed electron density by a plane perpendicular to a LOS with Carrington longitude of $325^{\circ}$ and latidude of $0^{\circ}$. The reconstruction for the period before CME2ab is shown on the left side while the reconstruction for the period after these CMEs is shown on right side. The east sides from the Sun correspond to the region with Carrington longitude of $235^{\circ}$, i.e. where the CME2ab took place. We see that the height of the streamer in the CME region after the CMEs is relatively slightly reduced in contrary with the CME1 case.

\section{Conclusions}

1. To our knowledge this is the first direct evidence (eliminated from the LOS effect) of the streamer blow out effect caused by the slow CME. Llebaria et al. (2006) made a statistical analysis of the interaction of CMEs with the streamer belt and have found that $72 \%$ cases of slow CMEs caused dimming in the streamer (i.e. steep decrease of the streamer brightness after the event) while this effect occured only for $18 \%$ of the investigated fast CMEs.

The height of the streamer in the CME region after the CME1 is significantly reduced in contrast to with the CME2ab case where this height remains almost the same as before as well after the CME2ab.

2. The potential magnetic field configuration in CME1 initiation region before the $\mathrm{CME}$ occured does not agree with the coronal density structure, while after the CME the agreement between the field and density is much better. This could be a manifistation of that the field before the CME1 is non-potential and after the CME1 the field relaxes towards a more potential state. Also, the interpretation of the position of heliospheric current sheet based on PFSS model during a pre-CME period could be very questionable.

On the other hand, for the fast CME2ab the PFSS model and the reconstructed streamer belt structure above the source region are in good agreement both before and after the
CME2ab occurred. On smaller scales inside the active region the PFSS model could not be validated. This could be indication of different initiation and/or propagation mechanisms for these two cases. Particularly, the source region for CME1 could be located higher in the corona than for CME2ab.

The present paper is a first step in the analysis of interactions of CMEs with the streamer belt structure using 3-D structure of the belt obtained from the tomographic reconstruction. We considered here only two cases. A 3-D reconstruction of the type discussed in this paper could be produced for almost every Carrington rotation during STEREO operational period in a robust way allowing a more systematic study.

\section{Appendix A}

\section{CME mass calculation}

We estimated the masses of CME2a and CME2b in the following way:

$m=\sum_{i} \frac{B_{\mathrm{obs}}\left(x_{i}, y_{i}\right)}{B_{\mathrm{e}}\left(x_{i}, y_{i}, \psi\right)} \cdot 1.97 \times 10^{-24} \mathrm{~g}$,

where the ratio of $B_{\mathrm{obs}}\left(x_{i}, y_{i}\right) / B_{\mathrm{e}}\left(x_{i}, y_{i}, \psi\right)$ is the excess number of electrons, $B_{\mathrm{obs}}\left(x_{i}, y_{i}\right)$ is the excess brightness observed in a given pixel with index number $i$ at location $\left(x_{i}, y_{i}\right)$ in the plane of the sky (POS), $B_{\mathrm{e}}\left(x_{i}, y_{i}, \psi\right)$ is the brightness of a single electron at that location at angle $\psi$ away from POS derived from the Thompson scattering equations (Billings, 1966). The angle $\psi$ at location $\left(x_{0}, y_{0}\right)$ can be computed by the following equations: $\psi=\operatorname{atan}\left(z_{0} / \sqrt{x_{0}^{2}+y_{0}^{2}}\right)$. The error in CME's mass estimation could be up to $50 \%$ (Vourlidas et al., 2000).

Acknowledgements. Thanks to Bernd Inhester for useful disscussions and comments that help to improve the paper. Also thanks to Gordon Petrie for usefull comments about potential field reconstruction methods. Thank to unknown referee for useful comments helped to improve the paper. This research was partially supported by NSF National Space Weather Program grant number AGS0819971.

Topical Editor R. Forsyth thanks F. Auchere and another anonymous referee for their help in evaluating this paper.

\section{References}

Altschuler, M. D. and Newkirk, G.: Magnetic Fields and the Structure of the Solar Corona. I: Methods of Calculating Coronal Fields, Solar Phys., 9, 131-149, 1969.

Billings, D. E.: A Guide to the Solar Corona, New York: Academic Press, 1966.

Davila, J. M.: Solar Tomography, Astrophys. J., 423, 871-877, 1994. 
Demoulin, P., Cuperman, S., and Semel, M.: Determination of force-free magnetic fields above the photosphere using threecomponent boundary conditions. II - Analysis and minimization of scale-related growing modes and of computational induced singularities, Astron. \& Astrophys., 263, 351-360, 1992.

Forbes, T. G.: A review on the genesis of coronal mass ejections, J. Geophys. Res., 105, 23153-23166, 2000.

Frazin, R. A. and Janzen, P.: Tomography of the Solar Corona. II. Robust, Regularized, Positive Estimation of the Threedimensional Electron Density Distribution from LASCO-C2 Polarized White-Light Images, Astrophys. J., 570, 408-422, 2002.

Frazin, R. A. and Kamalabadi, F.: Rotational Tomography For 3d Reconstruction Of The White-Light And Euv Corona In The Post-Soho Era, Solar Phys., 228, 219-237, 2005.

Hudson, H. S., Bougeret, J.-L., and Burkepile, J.: Coronal mass ejections: overview of observations, Space Sci. Rev., 123, 1330, 2006.

Jiao, L., McClymont, A. N., and Mikić, Z.: Reconstruction of the Three-Dimensional Coronal Magnetic Field, Solar Phys., 174, 311-327, 1997.

Judge, P. G.: Spectral Lines for Polarization Measurements of the Coronal Magnetic Field. V. Information Content of Magnetic Dipole Lines, Astrophys. J., 662, 677-690, 2007.

Klimchuk, J. A.: Theory of Coronal Mass Ejections, in: Space Weather (Geophysical Monograph 125), edited by: Song, P., Singer, H., and Siscoe, G., American Geophysical Union, 143, 2001.

Kramar, M. and Inhester, B.: Inversion of coronal Zeeman and Hanle observations to reconstruct the coronal magnetic field, Memorie della Societa Astronomica Italiana, 78, 120-125, 2007.

Kramar, M., Inhester, B., and Solanki, S. K.: Vector tomography for the coronal magnetic field. I: longitudinal Zeeman effect measurements, Astron. Astrophys., 456, 665-673, 2006.

Kramar, M., Jones, S., Davila, J., Inhester, B., and Mierla, M.: On the Tomographic Reconstruction of the 3D Electron Density for the Solar Corona from STEREO COR1 Data, Solar Phys., 259, 109-121, 2009.

Lin, H., Kuhn, J. R., and Coulter, R.: Coronal Magnetic Field Measurements, Astrophys. J., 613, 177-180, 2004.
Llebaria, A., Saez, F., Lamy, P., Robelus, S., and Boursier, Y.: Interactions of CMEs with the streamer belt, ESASP, 617, 135-138, 2006.

Mikic, Z. and Lee, M. A.: An introduction to theory and models of CMEs, shocks, and solar energetics particles, Space Sci. Rev., 123, 57-80, 2006.

Quemerais, E. and Lamy, P.: Two-dimensional electron density in the solar corona from inversion of white light images - Application to SOHO/LASCO-C2 observations, Astron. Astrophys., 393, 295-304, 2002.

Robbrecht, E., Patsourakos, S., and Vourlidas, A.: No Trace Left Behind: Stereo Observation of a Coronal Mass Ejection without Low Coronal Signatures, Astrophys. J., 701, 283-291, 2009.

Sakurai, T.: Computational modeling of magnetic fields in solar active regions, Space Sci. Rev., 51, 11-48, 1989.

Thompson, W. T. and Reginald, N. L.: The Radiometric and Pointing Calibration of SECCHI COR1 on STEREO, Solar Phys., 250, 443-454, 2008.

Tikhonov, A. N.: Solution of incorrectly formulated problems and the regularization method, Soviet Math. Dokl., 4, 1035-1038, 1963.

Tomczyk, S., Card, G. L., Darnell, T., Elmore, D. F., Lull, R., Nelson, P. G., Streander, K. V., Burkepile, J., Casini, R., and Judge, P. G.: An Instrument to Measure Coronal Emission Line Polarization, Solar Phys., 247, 411-428, 2008.

Van de Hulst, H. C.: The electron density of the solar corona, Bull. Astron. Inst. Netherlands, 11, 135-150, 1950.

Vourlidas, A., Subramanian, P., Dere, K. P., and Howard, R. A.: Large-angle spectrometric coronagraph measurements of the energetics of coronal mass ejections, Astrophys. J., 534, 456-467, 2000.

Wiegelmann, T.: Nonlinear force-free modeling of the solar coronal magnetic field, J. Geophys. Res., 113, A03S02, doi:10.1029/2007JA012432, 2008.

Wiegelmann, T., Lagg, A., Solanki, S. K., Inhester, B., and Woch, J.: Comparing magnetic field extrapolations with measurements of magnetic loops, Astron. Astrophys., 433, 701-705, 2005.

Zidowitz, S.: Coronal structure of the Whole Sun Month: A tomographic reconstruction, J. Geophys. Res., 104, 9727-9734, 1999. 